\title{
VISUALIZATION ASPECTS OF MOTION TRACKING AND ANALYSIS OF THE OUTER SURFACE OF THE LEFT VENTRICLE
}

\author{
Quatember $\mathrm{B}^{1}$, Recheis $\mathrm{W}^{1}$, Mayr $\mathrm{M}^{2}$, Demertzis $\mathrm{S}^{3}$, Allasia $\mathrm{G}^{4}$, De Rossi $\mathrm{A}^{4}$, Cavoretto $\mathrm{R}^{4}$, Venturino ${ }^{4}$, \\ De Lazzari $\mathrm{C}^{5}$ \\ ${ }^{1}$ Univ.-Klinik für Radiologie, Innsbruck Medical University, Austria \\ ${ }^{2}$ University of Applied Sciences Wiener Neustadt, Austria \\ ${ }^{3}$ Cardiocentro Ticino, Lugano, Switzerland \\ ${ }^{4}$ Dipartimento di Matematica, Universita degli Studi di Torino, Italy \\ ${ }^{5}$ Institute of Clinical Physiology, C.N.R., Rome, Italy \\ Bernhard.Quatember@uibk.ac.at
}

\begin{abstract}
The quantitative assessment of the motion and deformation of the heart is instrumental to diagnosis. We developed an accurate method for tracking and analysing the regional motion and deformation of the heart. To be of clinical value, the results must be visualized, and we paid much attention to all relevant visualization aspects.
\end{abstract}

Keywords: Heart wall motion, cardiology, multimodal imaging, differential geometry, hyperstreamlines

\section{Introduction}

Motion tracking and quantitative motion analyses have become an important investigative and diagnostic method and an important basis for the patient-specific planning of an effective therapy and the assessment of its success [1]. We will concentrate here on specific problems in the clinical domain of coronary artery disease where it is highly meaningful to acquire a fair knowledge of the heart wall motion, since important processes of disease manifest themselves in abnormal motions of the heart, especially of the left ventricle. Motion analysis will thus enable cardiologists to delve into greater diagnostic details. Unfortunately, the accuracy of all currently applied methods for the acquisition of the regional heart wall motion is rather limited. We developed a sufficiently accurate technique for tracking and analyzing the regional motion of the outer surface of the myocardium throughout the cardiac cycle which is based on cardiac CT and biplane coronary angiography. In doing so, we confined ourselves on the outer surface of the left ventricle.

\section{Methods}

Our motion tracking approach is based on multimodal cardiac imagery, viz. cardiac CT and biplane coronary angiography [2]. In the end-diastolic position, the epicardial surface in the 3D CT data is segmented and registered to the $3 \mathrm{D}$ skeleton representation of the coronary artery tree obtained from the end-diastolic cineangiographic frame. In doing so, a landmark-based approach with the use of TPS transformations has been chosen. The motion tracking is accomplished by carrying out further landmark-based TPS transformations of the surface to the successive frames of the cineangiogram. In each segmented transaxial slice of the CT, we define an appropriate number of nodes and construct a spline (NURBS) curve which approximates these nodes [3]. The subdivision points of all transaxial slices are regarded as nodes of a surface mesh. The nodes belonging to each of the transaxial slices are connected to each other with an interpolating spline (NURBS) curve which we call transversal contour curve. We also compute an array of longitudinal interpolating NURBS curves, each of which passes through corresponding points in all slices. These two arrays of NURBS curves constitute a surface mesh. For our motion tracking and analysis task, we take the surface obtained for the end of the diastole in the aforementioned manner and the skeletonized representations of the coronary artery tree derived from all cineangiographic time frames as a basis. Starting from the end-diastolic position, we co-register the surface step by step to the individual frames of the cineangiogram. We thus generate a deformable mesh to represent the geometry of the outer surface of the myocardium during motion. In the clinical assessment of the patient's regional variation of the heart wall motility, visualization techniques will play a decisive role. Our visualization efforts aim at an objective and insightful presentation of the local, regional and global motility of the myocardium. In Fig. 1(a), such a surface mesh belonging to the end-diastolic position can be seen, Fig. 1(b) shows the surface mesh belonging to the end-systolic position of the left ventricle. In both figures only the nodes of the mesh are depicted whereas, to avoid clutter, the spline curves are omitted.

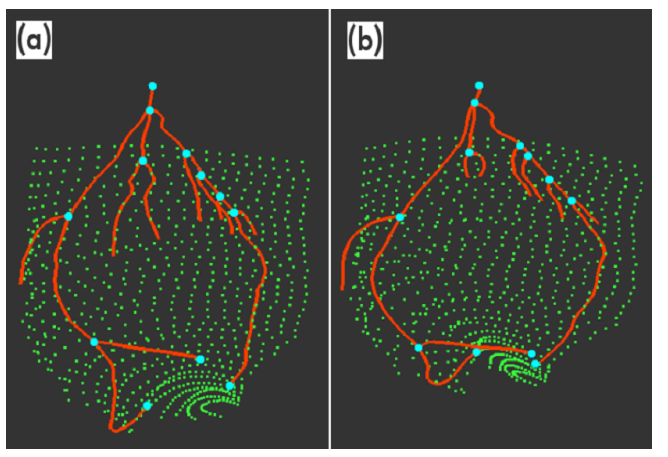

Fig. 1: Surface meshes representing the moving outer surface of the left ventricle: (a) at end-diastolic position;

(b) at end-systolic position.

The deforming surface mesh holds outstanding qualifications for the development of the aspired diagnostically conclusive 
visualization methods. We can easily construct trajectories for an arbitrarily chosen material point on the surface of the myocardium (i) by identifying the corresponding points of the deformable mesh within all time frames of the cineangiogram and (ii) by drawing a NURBS curve which fits this sequence of points (cf. Fig. 2).

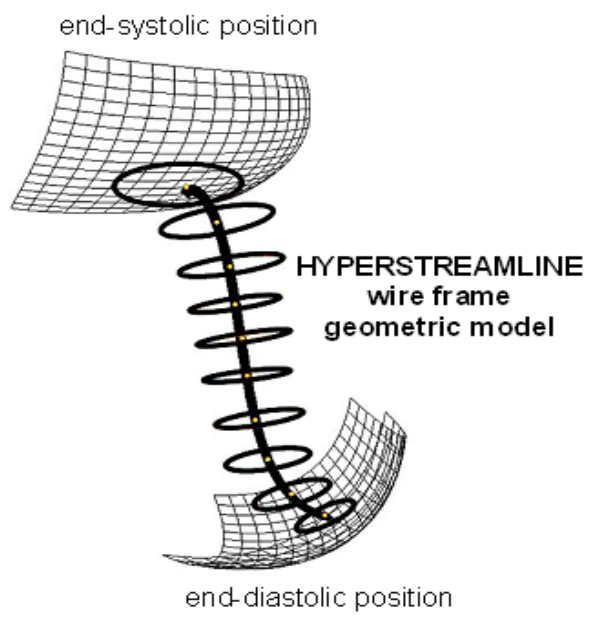

Fig. 2: Visualization of the motion and deformation of the left the ventricular surface during the cardiac cycle - wire frame geometric model of hyperstremline.

In each and every point along such a trajectory, the surface strain tensor can be calculated and visualized (cf. Fig. 2). Its visualization will allow cardiologists an objective assessment of the deformation of the moving myocardium. Our deformable mesh will also allow us to introduce further highly conclusive parameters based on differential geometry.

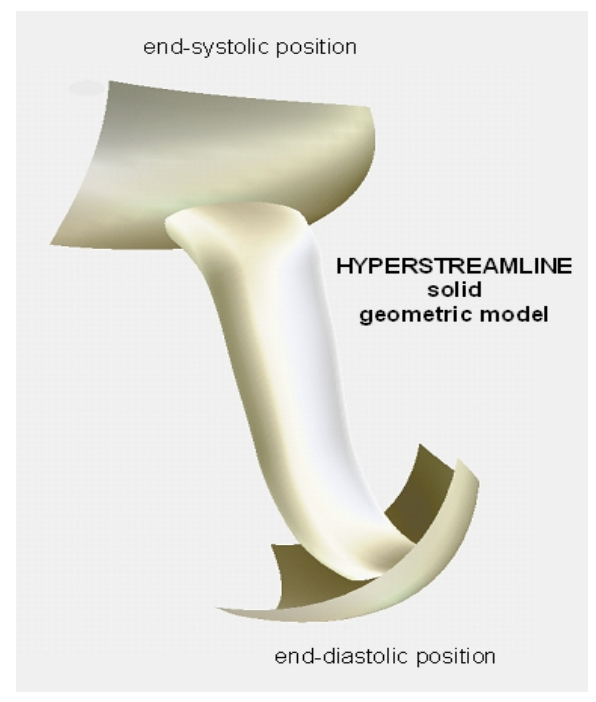

Fig. 3: Visualization of the motion and deformation of the left the ventricular surface during the cardiac cycle solid geometric model of hyperstremline.

In particular, we employ the metric tensor or the right Cauchy-Green deformation tensor, respectively, to describe the ventricular deformation and its variation during the cardiac cycle. We are able to calculate these tensor quantities for all points throughout the entire ventricular surface as well as their motion during the cardiac cycle. However, the computations result in a wealth of numerical data. To be of clinical value, the results must be represented in such a manner that cardiologists are able to use them in drawing diagnostic conclusions, especially in cases of regional motion abnormalities of the heart.

\section{Results}

As mentioned earlier, we obtained a family of surface meshes which describes the motion of the outer surface of the left ventricle between its end-diastolic and end-systolic positions and trajectories describing the motion of arbitrarily chosen points on the ventricular surface (Fig. 1 and Fig. 2). The deformation of the ventricular surface which has been described by means of Cauchy-Green deformation tensors is represented by a circle at the end-diastolic position and ellipses belonging to the end-systolic position and to a sufficiently large number of intermediate positions. As can be seen in Fig. 2, the circle which distorts into ellipses while sweeping along the trajectory forms the wireframe representation of a tubular structure which is called hyperstreamline. Thus, Fig. 2 depicts the wireframe representation of such a hyperstreamline by showing the individual cross sections of its tubular geometry, whereas in Fig. 3, a solid geometric model can be seen which has been derived from the wireframe model in Fig. 2.

\section{Discussion}

We aim at providing cardiologists with a software tool for carrying out quantitative investigations of the motion and deformation of the outer surface of the left ventricle during the cardiac cycle. We described this tool for diagnoses of regional abnormalities of cardiac motion caused by a reduced myocardial contractility in coronary artery disease [4].

\section{Acknowledgement}

The work described in this paper is partially supported by the "Austrian GRID" project, funded by the Austrian BMBWK (Federal Ministry for Education, Science and Culture) under contract GZ 4003/2-VI/4c/2004.

\section{Bibliography}

[1] Cheung Y-f.: The role of 3D wall motion tracking in heart failure, Nature Reviews Cardiology, vol. 9, pp. 644-657, 2012.

[2] Quatember B., Mayr M. et. al.: Geometric modeling and motion analysis of the epicardial surface of the heart. Mathematics and Computers in Simulation, vol. 81, pp. 608-622, 2010.

[3] L.A. Piegl, W. Tiller, The NURBS book, Second ed., Berlin: Springer; 1997.

[4] Tee, M., Noble, J.A. et. al.: Imaging techniques for cardiac strain and deformation: comparison of echocardiography, cardiac magnetic resonance and cardiac computed tomography, Expert Review of Cardiovascular Therapy, vol. 11, pp. 221-231, 2013. 